\title{
Smart Assistive Activity Recognition Device for Differently Abled People Based on Machine Learning - SAARD
}

\author{
Jayashree Agarkhed ${ }^{1, *}$ Lubna Tahreem ${ }^{2}$ \\ 1,2 Department of Computer Science \& Engineering, P.D.A College of Engineering, Kalaburagi, Karnataka. \\ ${ }^{*}$ Corresponding author. Email: lubnatahreem789@gmail.com
}

\begin{abstract}
Science and Technology have made human life addictive to comfort, yet concurrently there exists an oppressed gathering of individuals who are battling for tracking down a creative way that can make their life easier for them. After concentrating and highlighting the problems faced by the differently abled people like blind and deaf, solving it through a device alone is a very hard task. A ton of exploration has been done on every issue and arrangements have been proposed independently. Objective of the smart assistive device SAARD (Smart Assistive Activity Recognition Device for Differently Abled People) is to recognize activity for differently abled people so; they feel confident and independent by helping them to know objects surrounding them. The Proposed device SAARD help the differently abled people by taking images and give the output in form of audio. Along with that it also detects obstacles and surrounding sound which alert them.
\end{abstract}

Keywords: Alert, Assistive device, Audio, Buzzer, Differently abled, HAR, Vibration.

\section{INTRODUCTION}

Around 1300 million individuals have some sort of greatly reduced vision among which 18.85 crore individuals have a light blindness disability, 21,700 crore have medium to extreme vision blindness disability, 3.6 crore individuals are visionless and person beyond 50 years age have vision blindness [1]. India is estimated as home to the greatest amount of visually challenged individuals. According to WHO, among the total populace around $5 \%$ or 46.6 crore individuals are deaf.

The advancements in Science and Technology have reached to incredible statures in making the Human Life simpler and agreeable inside a limited ability to focus time. During the most recent decades, we desire to run over different innovations that have built our life so simpler and agreeable that even to perform a task we don't require to move portion of our body. Yet, continually running in the competition to be in front of everybody we have failed to recall that we actually have a segment of our populace called the truly incapacitated individuals who are denied of the headways of science and technology since it has not given them that solace that is needed by them to feel that they also are the piece of the common people and can walk together with each other [1].

Human Activity Recognition (HAR) refers to the task of measuring the physical activity of a person via the use of objective technology. This task is extremely challenging owing to the complexity and diversity of human activities. HAR is considered as one of the most promising assistive technology tools to support differently abled daily.

AI (Artificial intelligence) branch ML (Machine learning) which gives a framework which can naturally take in itself and improve automatically from its activities without being expressly programmed. AI is wide-going piece of programming stressed over building splendid machines fit for performing the piece of work that customarily require human understanding. The difference in lives of people the way they live in some or another way is due to advancement in recreated knowledge. Those progressions aren't simply advantageous - they assist us with imparting, access more things, and to live more autonomously. From language acknowledgment programming, to autonomous vehicle, to brilliant home innovation, to the mechanical progressions not too far off, $\mathrm{AI}$ is assisting 
those with in capacities. Somebody who has no inabilities may view at their Alexa or Echo as an advantageous method to look for a formula or play a tune, yet for somebody with a visual handicap, it's a piece of innovation that transforms them. Numerous individuals may consider AI and think about a world with robots and modern innovation, yet for those with handicaps, AI innovation opens up a universe of openness.

Internet of things (IoT) portrays the organization of actual articles - "things" or objects. IoT connects many objects to the Internet. It engages the exchanging of data never open, and brings customers the information in a much safer way. These technologies are used to develop the proposed device. Existing tools can't be utilized to process real time information in the world. The proposed device SAARD is designed for differently abled people so that they can use this device in real time. Every selective day might have issues while knowing and connecting using their current circumstance, especially the obscure [2].

The prior works on this system has issues like it is only for blind or deaf or dumb. The system which is built for all is used for communication purpose it only helps them to communicate with each other or others it doesn't help them with the surrounding environment objects so that they can go anywhere with confident. Many of the differently abled people are illiterate they can't read the text on screen which makes them feel independent on others.

The proposed device SAARD is used to help differently abled people by recognizing daily activity by giving them information about the surrounding environment. All the design and layout is done bearing in mind of differently abled people. First it will convert picture to text and then text to audio for the differently abled person who deserve to live independently by using You Only Look Once (YOLO) algorithm that goes through a variety of a very mind-boggling convolutional neural organization design called the darknet with OpenCV and google text to speech. The proposed device SAARD will continuously capture multiple frames using a camera interfaced on raspberry pi processor and the frames then converted to audio segment, the obtained results achieve the success of the proposed SAARD prototype in giving differently abled users the capability to understand unfamiliar surroundings by recognizing the surrounding activity, through an easily adaptable device with this profound object identification model.

The proposed device SAARD also helps to recognize the obstacles before the individual. When an obstacle comes near it is detected utilizing ultrasonic sensor at a distance of 5 meters then it signals to raspberry pi. The raspberry pi then alerts the person through buzzer. The proposed device SAARD also alerts the person by vibrating when it detects a sound to assist the differently able people like deaf so, that they can go anywhere anytime independently.

This paper is assembled in 5 sections; section 1 outlines the proposed device introduction Section 2 represents the related work. Section 3 represents proposed system architecture and algorithm of proposed System. System analysis and discussions are address in section 4. Conclusion is in Section

\section{RELATED WORK}

This section discusses various research work on machine learning and object detection to support differently abled people.

SHAROJAN BRIDGE the framework model of this framework [1] depicted the work to beat any issues during the time spent correspondence between the Blind, Deaf and Peaceful individuals. Extension SHAROJAN BRIDGE will employ Wearable Technology, Texas Arrangement Circuitry and Arduino circuit gatherings to be able to offer a method to talk to men and women who are special.

Brilliant Glass Vision are specific for the individual that outwardly impeded [2] talk whether it could find restrictions and measure miles utilizing ultrasonic and microcontroller. Close up to getting proportions from the environment, its miles sent to outwardly impeded individuals through headsets. The SIM900A GSM/GPRS (Global Systems for Mobile/ General Packet Radio Service) module is useful to gain data through the net. The swap is discovered with all the construction that is usually useful for crisis commitments like sending text, comprising moment, temperature and different regions controlled by typically the watchmen if they are deadened there can end up being a hazard. With using brilliant glass, out there of entryways could possibly be the person that may be frail can go walking the neighbourhood regarding indoor and out there of entryways. A great instrument which could no route may need to fast apparent inability in addition to be outfitted to offer orders to typically the applicable.

In [3] they introduced a route help for the visually impaired and the blind individuals. They plan and carry out a savvy cap which assists the visually impaired and blind individuals with exploring openly by encountering their environmental factors. The Tensor Flow API (Application Programming Interface) is widely used for object detection and trained using COCO dataset (Common Objects in Context). OpenCV helps in image processing operations and eSpeak performs the text to voice conversion The landscape over the man or perhaps lady can end up being caught the use regarding a NoIR digicam and the gadgets inside the scene may possibly be perceived. Typically, the earphones will 
communicate a sound ultimate product that exhibits the thing an individual have analysed.

The author in IOT based assistive device for deaf, dumb and blind people proposed a device that enables visually challenged people to read by taking an image. The raspberry $\mathrm{Pi}$ connects the camera, microphone, speaker and (Liquid-Crystal Display) LCD display. Further, Image to text conversion and speech synthesis is done, converting it into an audio format that reads out the extracted text translating documents, books etc. Google Cloud Vision API (Application Programming Interfacing) is used to classify images, optical character recognition (OCR) is used to detect text within images. Initially Conventional neural network (CNN) based model is used to detect localized lines of text and generates a set of bounding boxes. For deaf, it takes the speech as input through microphone and recorded audio is converted to text and displayed as pop up window on screen. Speech to text conversion is done through Google cloud Speech. For dumb it takes input as text through keyboard and convert the text to speech through Google Text to Speech API (gTTS API) [4].

The HospiSign period framework allows inside clients to convey the reason behind a small while by responding between 4 in order to six inquiries that will envelop a person PC, somewhat demonstrated to find out client prepared questions and plans, along with a Microsoft Kinect v2 sensor. These people should be attempted as far because correspondence determined by $\mathrm{PC}$ vision via an arranged up development verification system to assist clients whose spending attention to bad luck in a problems clinical climate offers been made. Difficult of hearing customers can make use of their own marks to speak using the gadget, which usually presents a made up record of the particular exchange. A blend of hand job plus movement capacities exhibited with the Temporary Template and called through the Arbitrary Decision Forest produced probably the most straightforward results [5].

In [6] authors proposed a non-vision based idea that will assist in removing or at least reducing this gap between the speech impaired and the able-bodied people. The prototype involves Raspberry pi, Arduino nano as an A/D converter which are interfaced with flex sensors and accelerometer for reading the hand gestures, speaker module, (Organic Light-Emitting Diode) OLED, hand glove and a power supply of $5 \mathrm{v}$. When a specific sign is made, the flex sensors gets bent and unique values are generated. These values are stored in the database and when the sign is made the microcontroller matches the values with the stored values and the output is made available in the form of text on the OLED and an audio from the speaker which is connected to the raspberry pi.

Maker of an Implementation of a new methodology for personal communication system using raspberry-pi
[7] proposed a technique for a blind person to read a text or paragraph, and it can be provided by scanning an image through Logitech camera which converts a scanned picture to text through Tesseract OCR (online character recognition) OCR technique, the adaptive linear threshold techniques are used to change the picture into binary mapped images text is read out by the e-speak through the speaker. Image is scanned, cropped and converted to a grayscale and then blur the resized picture by using the gaussian blur threshold function to get the concentrated area of the picture. After identifying the angle and contours between 2 fingers. Count the number of angles which is less than ninety degrees and identify the number of defects. According to the total number of defects, the message or text is displayed, for the deaf people it facilitates a way to read a text by voice or speech-to-text conversion (STP) and it also provides a technique for dumb people using text-to-voice conversion through which blind people are able to read the words using Tesseract OCR (online character recognition), online and dumb people communicate through to text which is read out by espeak.

The hand glove is fitted with Flex sensors on the length of every finger. These Flex sensors and Accelerometer are connected to the analog to digital converter (MCP3008) and the output of MCP3008 is given to the Raspberry Pi. When the required gesture is made the flex sensors provide the output within the kind of variable resistance that varies with degree of bend. This output is given as input to the MCP3008, which performs analog to digital conversion and gives the output to the Raspberry Pi. Raspberry Pi processes the data and the corresponding output is displayed on liquid crystal display, at the same time a speech output is played through speaker. The speech will be processed and the output in textual form is displayed on LCD [8].

Inside [9] examined different noticeable replacement buildings which have recently been created recently. He or she additionally intends to be able to present a method that re-establishes typically the critical factor regarding the apparent device to the identifiable proof of encompassing things. The strategy depends upon video check and interpretation. Within this way, there is a commitment in order to uncover a visible replacement machine dependent absolutely at the particular assessment of the quick and amazing set up of rules with regard to distinguishing and finding contraptions in image.

Eye improvement appraisal is another divided way to cope with the adjustment associated with action in [10] that will show eye development data recorded the particular utilization of the electrooculography (EOG) framework. This individual initially portrayed plus assessed estimations in order to recognize 3 credit of eye development from EOG indicators - saccades, 
obsessions, and flashes -- and proposed techniques for looking at situations of obscured vision improvement. By then, create 90 precise brilliant lights that depend on those ascribe and choose the part that utilizes one of the most un-comprehensive bumbles thorough of assurance (mRMR).

A knowledge-driven approach to real-time, persistent movement acknowledgment dependent on multi sensor information streams in smart homes has clarified important aspects in [11]. They dissect the qualities of smart homes and Activities of Daily Living (ADL) whereupon they fabricated both setting and ADL ontologies. They additionally present a conventional framework design for the proposed information driven approach and depict the fundamental ontology based recognition process.

In [12] they portrayed item identification as a relapse issue for hop bins with spatial in addition to related polish leads. Singular neural business predicts hop bins and complexity likelihood on the twice through the whole photo within a solitary examination. As opposed to the top step - in: class revelation construction, (You only look once) YOLO commits added limiting errors, in any case is extra cautious to count on main positives.

The [13] additional non - unmistakable discussion by indicates of sign conversion which has already been accomplished for the particular declaration of train route lines within India. For textual content interpretation, the utilization associated with successful and eco-friendly articulation - dependent absolutely techniques associated with rule -- based interpretation methodologies have been used that produce perfect yield with the particular guide of reducing repetition and therefore the particular translation season of joints and chains that help whole human - like edge parts.

Deaf Sign Language using Automatic Hand Gesture Robot Based on Microcontroller System [14] discussed the design and implementation of deaf sign language using automatic hand gesture robot using Bluetooth and Arduino board. Gesture controlled robot works on principle of flex sensors which records hand movement and sends data to the Arduino the information is transferred to Bluetooth. On the receiving end, the information is received via Bluetooth then passed to the microcontroller which takes various decisions based on the received information. Arduino also makes the decision such as displays on Liquid Crystal Display (LCD) with alphabet and executes sound.

The author designs the system with Python and Tensor Flow models to create a classifier on Raspberry Pi. They used a model called ssdlite_mobilnet_v2_coco to detect 9 different objects which could present on sidewalks. Combination of auditory and tactile senses to alert the user. A system called eSpeak is used to alert the user via headphones by reading the name of the detected object. At the same time, 3 different vibration sensors are used. When an obstacle was detected inside one of the predefined bounding boxes, the related vibration sensor was activated with the name of the object [15].

An android application that recognizes objects using real time object and text detection by scanning them [16]. The app doesn't need any photograph to detect the object. To enable robust recognition, first segment the object from the background using tensor flow machine learning API based on iterative diagram cuts. Then formulate the recognition problem as an instance retrieval task and the user gets to know the object through a text to speech method.

A transfer learning-based deep learning method is used in [17]. The 5 different convolutional neural networks $(\mathrm{CNN})$ are used. For the improvement in the result, the majority voting scheme is used. The CUB200-2011 dataset is used to detect different types of birds.

Survey focuses on critical role of machine learning in developing HAR applications based on inertial sensors in conjunction with physiological and environmental sensors. Activity recognition algorithms based on ML and DL are becoming central in HAR. They discussed datasets primarily in the literature, emphasizing publicly available datasets. Also presented a description of the recognition models most used in HAR. For this purpose, they have presented the most widely used DL and ML models and their results, both from the point of view of quality (accuracy) and quantity (number of recognized activities). And concluded HAR researchers still prefer classic ML models, mainly because they require a smaller amount of data and less computational power than DL models. However, the DL models have shown higher capacity in recognizing many complex activities [18].

A simple and effective Single-Shot Alignment Network (S2A-Net) for oriented object detection in aerial images [19]. With the Feature Alignment Module and Oriented Detection Module, there S2A-Net realizes full feature alignment and alleviates the inconsistency between regression and classification. Besides, they explore the approach to detect on large-size images for better speed-accuracy trade-off.

From the analysis of literature survey there are many assistive devices for differently abled people either for all or only for a particular disability but among them none of them have provided assistive device for helping them about recognizing the activity of surrounding objects or obstacles some has provided it but only a particular disability not for all disabled people [20-23]. 


\section{PROPOSED SYSTEM ARCHITECTURE}

The main motive of proposed device SAARD is to introduce an idea that can help the differently abled people by providing the surrounding information by detecting the activities like what objects are there around them or any obstacle is there on their way so, that they can go anywhere without being dependent on others. This section includes system architecture, algorithm of the proposed device SAARD and algorithm [24-27].

\subsection{Block Diagram}

The block diagram of proposed SAARD device is shown in figure 1 . In phase 1 first it takes the input image from camera at 45 frames-per-second and then divide the input image into a grid cell of $\mathrm{S} x \mathrm{~S}$. In phase 2 the model is trained with the Common Objects in Context (COCO) dataset. Image classification and localization are applied on each grid. While classifying each grid (You only look once) YOLOv3 predicts the bounding boxes based on the center of object and their corresponding class probabilities for objects and identify the object using class labels from the trained COCO dataset. If it predicts more than one bounding box for same object, then it uses Intersection over Union (IOU) and Non-Max Suppression on that object and gives the final or one bounding box for that object by calculating its confidence score so that we don't end up predicting multiple bounding boxes of same object. Finally, in phase 3 it detects object with bounding box along with the confidence score. The output is given as soon as object is detected it is converted to speech, the text description is then send to the Google Text-to-Speech API using the gTTS package and the speech output is given through headphones. If an object is detected using ultrasonic sensor at a distance of 3 meters then it signals to microcontroller. The microcontroller then alerts the person through alerter. The Proposed device SAARD also detects the surrounding sound through sound sensor and alerts the person through vibration which helps them through their daily lives.

The device has one raspberry pi board, camera, headphones, ultrasonic sensor, buzzer, sound sensor, vibration motor. The working of the proposed device SAARD starts by suitably powering the raspberry pi processor. In this way, the digicam is connected through one of the USB ports of the raspberry pi is instated. Genuine - time video is worried about a Logitech advanced camera that is then changed over into one edge. Wherein a particular thing is identified, it figures the self-conviction stage for each thing and refinement name and passes it to the raspberry pi processor.

By utilizing literary substance - to - discourse converter programming, printed content records like class marks, appraisals, etc. Are changed over into sound yield. Headphones identified with the sound jack of the raspberry pi offer sound insights relating to the article in the picture.

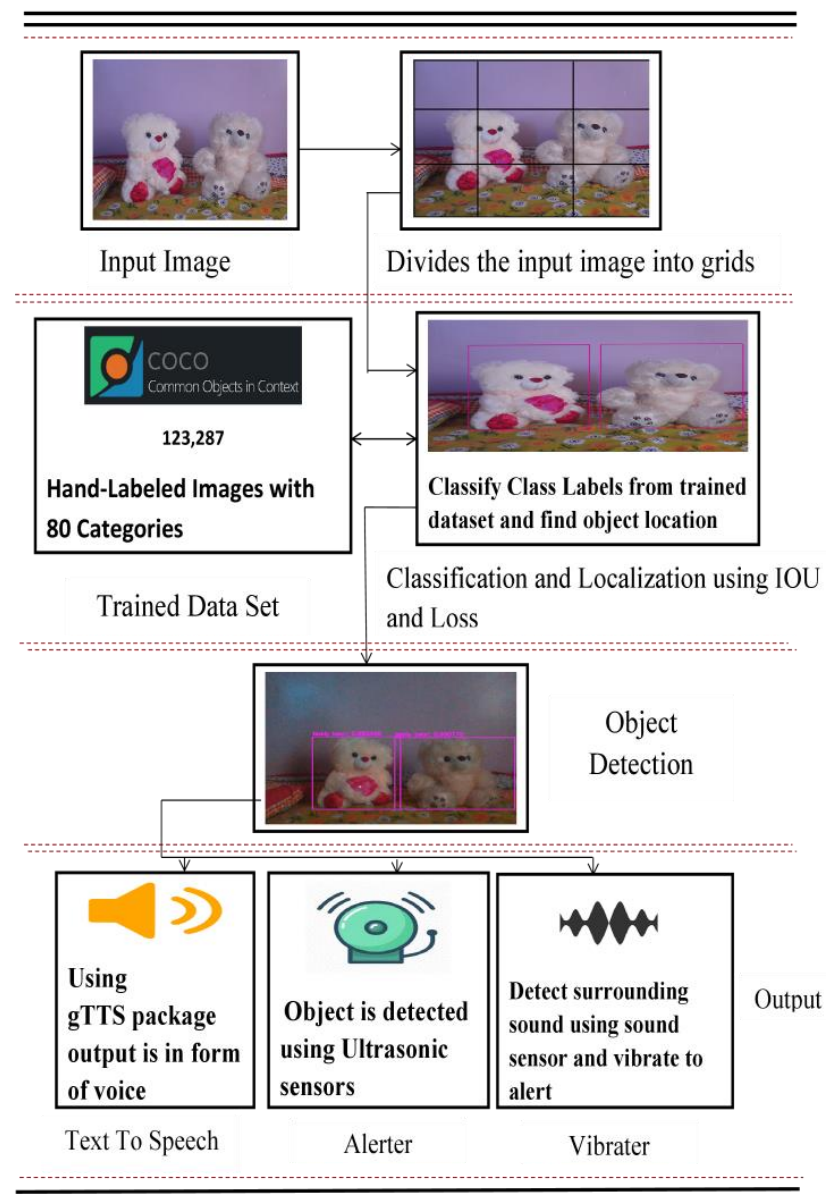

Figure 1 Block diagram of proposed device SAARD.

\subsection{Proposed Algorithm}

The Algorithm of Proposed device SAARD is shown below:

Step 1: Initialization: input image from the camera. while True:

if count $==0$ else:

frame_count $+=1$

\# Capture frame-by-frame

Step 2: Input image is divided into grids $13 \times 13$.

Step 3: bounding box around object is created

Step 3.1: \# Get the bounding box coordinates.

bx, by = boxes[i][0], boxes[i][1]

bw, bh = boxes[i][2], boxes[i][3]

Step 3.2: \# Draw the bounding box rectangle and label on the image.

cv.rectangle (img, (bx, (by), (bx+bw, by+bh), color, 2)

text $="\{\}:\{: 4 \mathrm{f}\} "$.format(labels[classids[i]], confidences[i])

Step 3.2: Get the box confidence score. scores $=$ detection $[5:]$ 
classid $=$ np.argmax $($ scores $)$

confidence $=$ scores $[$ classid]

Step 4: Consider only the predictions that are above a certain confidence level

if confidence > tconf:

box $=\operatorname{detection}[0: 4] * n p . \operatorname{array}([$ width, height, width, height])

centerX, centerY, bwidth, bheight $=$ box.astype('int')

Step 5: using the center $\mathrm{x}, \mathrm{y}$ coordinates to derive the top and the left corner of the bounding box

$$
\mathrm{X}=\operatorname{int}(\text { centerX }-(\text { bwidth / 2) })
$$$$
\mathrm{y}=\text { int }(\text { centerY }-(\text { bheight / 2) })
$$

Step 6: Append to list boxes.append([x, y, int(bwidth), int(bheight) $])$ confidences. append(float(confidence)) classids.append(classid)

Step 7: Generate the boxes, confidences, and classIDs

Step 8: Apply Non-Maxima Suppression to suppress overlapping bounding boxes idxs = cv.dnn.NMSBoxes(boxes, confidences, FLAGS.confidence, FLAGS.threshold)

Step 9: Draw labels and boxes on the image img = draw_labels_and_boxes(img, boxes, confidences, classids, idxs, colors, labels)

Step10: object detected and output is given as audio then go to step 13

Step11: if obstacle is detected it alerts by buzzer and go to step 13

Step12: if Sound is detected it vibrates and go to step 13 Step13: End

At step 1 the input image is initialized or taken from camera if count is 0 there is no image else if count is 1 , it captures 45 frames per sec. Next divide image into $13 \times 13$ grid cells. Each grid cell has bounding box coordinates values bx, by, bw, bh. rectangle bounding box with label on images as class id is taken. Then it takes the confidence score and consider only confidence score that is greater than 0.5 . The next step is to derive the top and left co-ordinates of bounding box using the center co-ordinates. It is compared with the list of class id present in dataset. Bounding box with class id and confidence score is created but, sometimes it creates more bounding boxes for a single object so, to remove unwanted bounding boxes non max suppression is done once the bounding box is created. Finally, we get the object with bounding box, class id and confidence score. Output is form of audio that there is object. The further Step shows that if there is obstacle on the way it alerts through a buzzer. It also detects surrounding sound and vibrates so, that differently abled people like deaf didn't get into an accident in case they didn't see a vehicle.

\section{SYSTEM ANALYSIS AND DISCUSSION}

The proposed device SAARD is compared with the existing systems by checking number of end services supported by each system and a conclusion is drawn that the proposed system tends to provide service that are not provided by other systems. All the existing system help differently abled people by means of communication to help them communicate with each other or with others and some systems provide services only to individual disability person but our proposed device SAARD help them to live independent of others and be confident to go anywhere anytime. It helps by recognizing activities to differently abled people by giving information about the surrounding object and also alert them if any object is detected or by detecting the surrounding sound and alert them through buzzer and vibration.

The Figure 2 shows how different algorithms take the image frames per sec. Our proposed method YOLOv3 take 45 frames per sec and is high comparative to others such as CNN takes 7 frames per sec. SIFT/SURF takes 23 frames per sec, SVM takes 30 frames per sec.

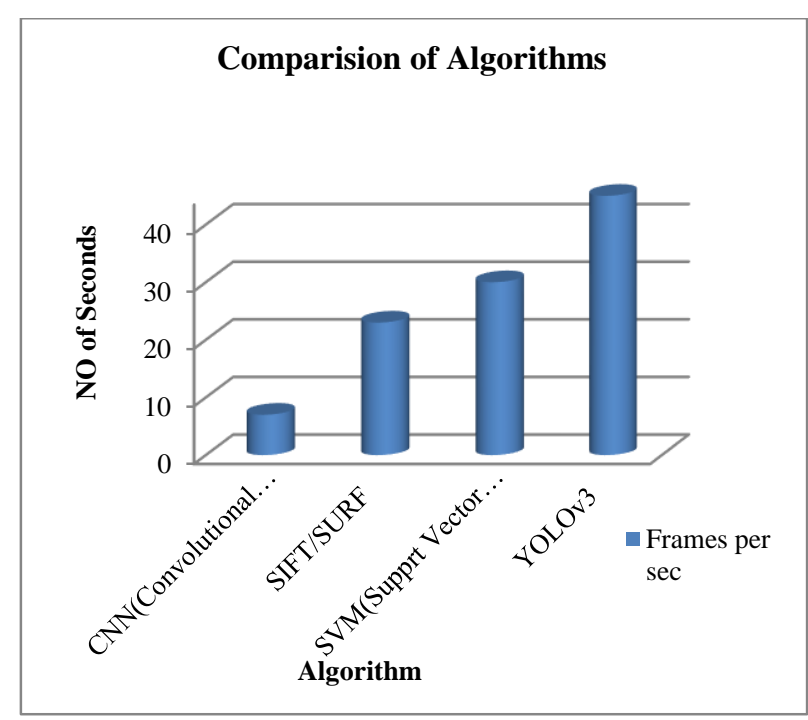

Figure 2 Comparison of different algorithms based on Frames.

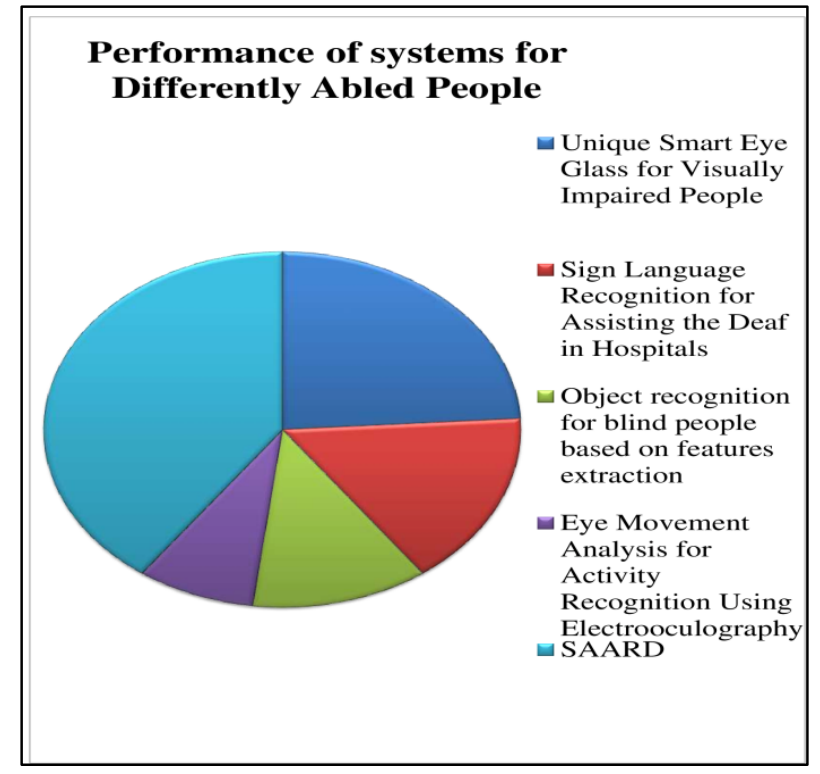

Figure 3 Performance of SAARD over different systems. 
As shown in Figure 3 above the performance of SAARD is greater than other systems as the proposed device SAARD provide accuracy greater than other systems and the percentage of efficiency provided by SAARD in terms of service is more and different from others as shown in Fig 4.

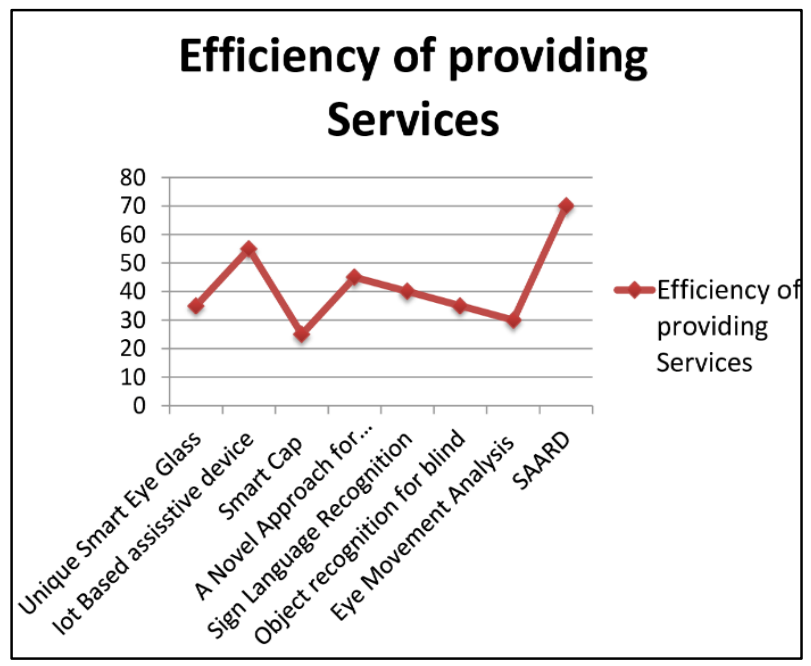

Figure 4 SAARD with approximately $70 \%$ efficiency

As some systems provide service to only particular type of disability and some systems provide service to differently abled people but to help them to communicate with other but, the SAARD not only help to recognize activities to differently abled people by giving information about surrounding objects but also help them to know surrounding sounds so that they don't get into accidents while crossing or walking on roads. It also helps them to detect the obstacle on their way if any and alert them, so they won't fall on ground and hurt themselves and can go anywhere without depending on others [28-30].

The SAARD device is compared with the existing devices by checking number of end services supported by each device and a conclusion is drawn that the SAARD device tends to provide the majority of end services and helps differently abled people to move from one place to another without any support of others.

The table 1 shown below shows the performance of existing system over SAARD device and how SAARD performance is better than other device [31-33].

By seeing the table 2 the no of services provided by SAARD is more than other devices so, the efficiency for no services provided by SAARD is greater than it not only helps to detect activities of objects but also detect obstacle, sounds and convert text to speech, image to speech, audio to audio. So, the differently able can live their life somewhat easy.

Table 1. Comparison of Performances of different assistive devices.

\begin{tabular}{|c|c|c|c|c|c|c|}
\hline References & Algorithm/classifiers & Results & Classes & Limitation & $\begin{array}{l}\text { Frames } \\
\text { per sec }\end{array}$ & Accuracy \\
\hline $\begin{array}{l}\text { Md. Razu } \\
\text { Miah, Md. } \\
\text { Sanwar } \\
\text { Hussain[2] }\end{array}$ & $\begin{array}{l}\text { NaiveBayes, } \\
\text { NeareastNeighbor } \\
\text { Decision Tree } \\
\text { LibSVM. }\end{array}$ & $\begin{array}{lrr}\text { Smart } & \text { glass for } \\
\text { visually } & \text { impaired } \\
\text { people. } & \end{array}$ & $\begin{array}{l}40 \\
\text { Features/ } \\
\text { Activities }\end{array}$ & $\begin{array}{l}\text { They are not as } \\
\text { accurate as vision- } \\
\text { based approaches. } \\
\begin{array}{lr}\text { The } & \text { outward } \\
\text { appearance } & \text { isn't } \\
\text { captured } & \end{array}\end{array}$ & - & $\begin{array}{l}\text { Intra-Subject } \\
\text { Validation = } \\
96.16 \% \\
\text { Intra-Subject } \\
\text { Cross Session } \\
\text { Testing = } \\
85.24 \%\end{array}$ \\
\hline $\begin{array}{l}\text { Nishajith.A, } \\
\text { Nivedha.J, } \\
\text { Shilpa.S.Nair, } \\
\text { Proff } \\
\text { Mohammed } \\
\text { Shaffi J.[3] }\end{array}$ & $\begin{array}{ll}(\mathrm{CNN}), & \\
\text { Convolutional } & \text { Neural } \\
\text { Network } & \text { model } \\
\text { TensorFlow } & \text { API, } \\
\text { open-source library. }\end{array}$ & $\begin{array}{ll}\text { Visual } & \text { Guidance } \\
\text { System. } & \end{array}$ & $\begin{array}{l}90 \\
\text { objects }\end{array}$ & $\begin{array}{l}\text { Detects only } 90 \\
\text { classes of objects. }\end{array}$ & 7 & $\begin{array}{l}\text { Accuracy } \\
\text { results are not } \\
\text { mentioned. }\end{array}$ \\
\hline $\begin{array}{l}\text { Necati Cihan } \\
\text { Camgoz, } \\
\text { Ahmet Alp } \\
\text { Kindiroglu } \\
\text { and Lale } \\
\text { Akarun.[5] }\end{array}$ & $\begin{array}{l}\text { Dynamic Time } \\
\text { Warping (DTW), k- } \\
\text { Nearest Neighbour's } \\
(\mathrm{k}-\mathrm{NN}) \text { algorithm. }\end{array}$ & $\begin{array}{l}\text { Sign Language } \\
\text { Recognition }\end{array}$ & 6 users & 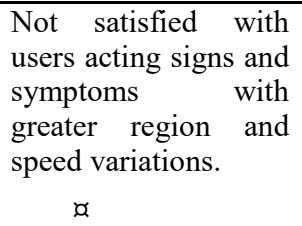 & - & $97.88 \%$. \\
\hline $\begin{array}{l}\text { Haney } \\
\text { Jabnoun, } \\
\text { Faouzi } \\
\text { Benzarti,and } \\
\text { Hamid } \\
\text { Amiri.[9] }\end{array}$ & $\begin{array}{lr}\text { Scale } & \text { Invariant } \\
\text { Features } & \text { Transform } \\
\text { approach } & \text { (SIFT), } \\
\text { Speeded Up } & \text { Robust } \\
\text { Features (SURF). }\end{array}$ & Object Recognition. & 1 user & $\begin{array}{l}\text { Improving the rate of } \\
\text { recognition. } \\
\text { Adding an auditory } \\
\text { translation for } \\
\text { identified objects. }\end{array}$ & 23 & $\begin{array}{c}\text { SIFT }=82 \% \\
\mathrm{SURF}=18 \%\end{array}$ \\
\hline
\end{tabular}




\begin{tabular}{|c|c|c|c|c|c|c|}
\hline $\begin{array}{l}\text { Andreas } \\
\text { Bulling, } \\
\text { Jamie A. } \\
\text { Ward, Hans } \\
\text { Gellersen, } \\
\text { and } \\
\text { Gerhard.[10] }\end{array}$ & $\begin{array}{lr}\text { Support } & \text { vector } \\
\text { machine } & \text { (SVM) } \\
\text { approach. } & \end{array}$ & $\begin{array}{l}\text { Activity Recognition } \\
\text { using } \\
\text { Movement. }\end{array}$ & $\begin{array}{l}90 \\
\text { Features }\end{array}$ & $\begin{array}{l}\text { Challenge to define a } \\
\text { single identifiable } \\
\text { activity. } \\
\text { Browsing the net is } \\
\text { more hard due to the } \\
\text { one-of-a-kind eye } \\
\text { actions available }\end{array}$ & 30 & $76.1 \%$ \\
\hline $\begin{array}{l}\text { Jayashree } \\
\text { Agarkhed, } \\
\text { Lubna } \\
\text { Tahreem }\end{array}$ & $\begin{array}{l}\text { CNN, YOLOv3, } \\
\text { COCO model. }\end{array}$ & $\begin{array}{l}\text { Provide } \\
\text { information about } \\
\text { surrounding } \\
\text { objects and detect } \\
\text { obstacles and } \\
\text { surrounding sound } \\
\text { for differently } \\
\text { abled people }\end{array}$ & $\begin{array}{l}1000 \text { or } \\
\text { more } \\
\text { features/ } \\
\text { objects }\end{array}$ & $\begin{array}{l}\text { If object has less } \\
\text { accuracy it ignores } \\
\text { the object. }\end{array}$ & 45 & $98 \%$ \\
\hline
\end{tabular}

Table 2. Comparison of no of services.

\begin{tabular}{|l|l|l|l|l|l|l|l|}
\hline No of Services & $\begin{array}{l}\text { Unique } \\
\text { Smart Eye } \\
\text { Glass }\end{array}$ & $\begin{array}{l}\text { Iot } \\
\text { Based } \\
\text { Assistive } \\
\text { Device }\end{array}$ & Smart cap & $\begin{array}{l}\text { Novel } \\
\text { Approach } \\
\text { sharojan } \\
\text { Bridge }\end{array}$ & $\begin{array}{l}\text { HospiSign } \\
\text { Sign } \\
\text { Language }\end{array}$ & $\begin{array}{l}\text { Object } \\
\text { Recognition }\end{array}$ & SAARD \\
\hline Object detection & $\checkmark$ & & $\checkmark$ & & & $\checkmark$ & $\checkmark$ \\
\hline Sending Sms & $\checkmark$ & & & & & & $\checkmark$ \\
\hline Text to speech & & $\checkmark$ & & $\checkmark$ & & & \\
\hline Speech to text & & $\checkmark$ & & $\checkmark$ & & & $\checkmark$ \\
\hline Audio to Audio & & & & $\checkmark$ & & & $\checkmark$ \\
\hline Image to speech & & & $\checkmark$ & & $\checkmark$ & & $\checkmark$ \\
\hline Hand Gesture & & & & & & & $\checkmark$ \\
\hline Obstacle Detection & & & & & & & \\
\hline Sound Detection & & & & & & & \\
\hline
\end{tabular}

\section{CONCLUSION}

SAARD proposed now not the simplest cognizance at the development and facilitation of other those who are different, but additionally compact and saves resources. The advanced technologies and habits make this device transportable, adaptable and convenient. The equipment proposed by using SAARD can be a high quality help in dealing with a number of the demanding situations faced by means of distinctive areas. To raise the task, the device can be made extra compact so that it is simple for users to use. The limitation is it doesn't have sign language support as it requires separate database and algorithm but, for future work we can use this and make device more effective. Another limitation is it doesn't recognize traffic signal lights and signs because it should be trained more to recognize the picture while pattern matching techniques also changes here and also require separate database. Can also extend this limitation to future work and can further improve device by giving audio directions whether to go right or left if there is an obstacle on way so that they can walk more freely.

\section{REFERENCES}

[1] Nishajith.A, Nivedha.J, Sha.S.Nair, Prof. Mohammed Shaffi.J, "Smart Cap - Wearable
Visual Guidance System For Blind", (ICIRCA 2018) IEEE Xplore

[2] Md. Razu, Md. Sanwar Hussain "A Unique Smart Eye Glass for Visually Impaired People", International Conference on Advancement in Electrical and Electronic Engineering 22-24 November, 2018, Gazipur, Bangladesh.

[3] Nishajith.A, Nivedha.J, Shilpa.S.N, Shaffi.J, "Smart Cap - Wearable Visual Guidance System For Blind", (ICIRCA 2018) IEEE Xplore.

[4] Karmel A, Anushka S, Muktak P, Diksha, "Iot Based assistive device for deaf, dumb and blind people", ICRTAC 2019.

[5] Necati Cihan, Ahmet Alp K and Lale Akarun "Sign Language Recognition for Assisting the Deaf in Hospitals" Conference Paper in Lecture Notes in Computer Science. Oct2016.

[6] S.Shareef, M.M Hussain, A.Gupta, Aejaz A, "Hand Gesture Recognition System for Deaf and Dumb", International Journal of Multidisciplinary and Current Educational Research (IJMCER) 2020.

[7] Yogeesh M, Sivakumar B "Implementation Of A New Methodology For Personal Communication System Using Raspberry-Pi”, International Journal of Recent Scientific Research, July, 2019. 
[8] Pavithra. K, Likhitha. M. S, Niranjan. S. D, Chandrakanth. S, Tejaswini. M. L, "Smart Chiro for Deaf and Dumb”, IJESC, August 2020.

[9] Hanen J, Benzarti and Hamid A, “Object Recognition For Blind People Based On Features Extraction", IEEE Conference 2014 IPAS'14. 9781-4799-7069-8/14/\$31.00 c2014 IEEE.

[10] Andreas, Jamie A Ward, Gellersen, and Gerhard T, "Eye Movement Analysis for Activity Recognition Using Electrooculography", IEEE. 00000000/00\$00.00 c2010 IEEE.

[11] L. Chen, Nugent and Wang, "A Knowledge-Driven Approach to Activity Recognition in Smart Homes", IEEE.

[12] Joseph, Santosh, Ross.G, Farhadi, "You Only Look Once: Unified, Real-Time Object Detection”, 2016 IEEE Conference.

[13] Farahanaaz Shaikh, Shreya Darunde, Nikita Wahie and Swapnil Mali, "Sign Language Translation System for Railway Station Announcements", 2019 IEEE Conference (IBSSC).

[14] Hay Man Oo, Khin Thandar Tun, May Lwin Thant, "Deaf Sign Language using Automatic Hand Gesture Robot Based on Microcontroller System", International Journal of Trend in Scientific Research and Development (IJTSRD) Volume 3 Issue 5, August 2019

[15] Sude Pehlivan and Mazlum Unay, Aydin Akan, "Designing Obstacle Detection and Alerting System for Visually Impaired People on Sidewalks", 2019 Medical Technologies

[16] Md. Amanat Khan Shishir, Shahariar Rashid Fahim, Fairuz Maesha Habib, Tanjila Farah, "Eye Assistant Using mobile application to help the visually impaired", (ICASERT 2019), 978-1-72813445-1/19/\$31.00 @2019 IEEE.

[17] Ms Bulbul Bamne, Lokesh Parashar, Upendra Singh, Neha s, "Transfer learning-based Object Detection by using Convolutional Neural Networks", (ICESC 2020) IEEE Xplore Part Number: CFP20V66-ART;

[18] Florenc D, Graziano P, Azra And Parisa, "Human Activity Recognition Using Inertial, Physiological and Environmental Sensors: A Comprehensive Survey", DOI:10.1109/ACCESS.2020.3037715.

[19] Jiaming Han, Jian Ding, Jie Li, Gui-Song Xia, "Align Deep Features for Oriented Object Detection”. arXiv:2008.09397v3 [cs.CV] 12 Jul 2021.

[20] Kumar, M. Keerthi, B. D. Parameshachari, S. Prabu, and Silvia liberata Ullo. "Comparative Analysis to Identify Efficient Technique for Interfacing BCI System." In IOP Conference
Series: Materials Science and Engineering, vol. 925, no. 1, p. 012062. IOP Publishing, 2020.

[21] Subramani, Prabu, K. Srinivas, R. Sujatha, and B. D. Parameshachari. "Prediction of muscular paralysis disease based on hybrid feature extraction with machine learning technique for COVID-19 and post-COVID-19 patients." Personal and Ubiquitous Computing (2021): 1-14.

[22] Do, Dinh-Thuan, Tu Anh Le, Tu N. Nguyen, Xingwang Li, and Khaled M. Rabie. "Joint impacts of imperfect CSI and imperfect SIC in cognitive radio-assisted

NOMA-V2X communications." IEEE Access 8 (2020): 128629. 128645 .

[23] Le, Ngoc Tuyen, Jing-Wein Wang, Duc Huy Le, Chih-Chiang Wang, and Tu N. Nguyen. "Fingerprint enhancement based on tensor of wavelet subbands for classification." IEEE Access 8 (2020): 6602-6615.

[24] Do, Dinh-Thuan, Tu Anh Le, Tu N. Nguyen, Xingwang Li, and Khaled M. Rabie. "Joint impacts of imperfect CSI and imperfect SIC in cognitive radio-assisted

NOMA-V2X communications." IEEE Access 8 (2020): 128629128645 .

[25] Subramani, Prabu, K. Srinivas, R. Sujatha, and B. D. Parameshachari. "Prediction of muscular paralysis disease based on hybrid feature extraction with machine learning technique for COVID-19 and post-COVID-19 patients." Personal and Ubiquitous Computing (2021): 1-14.

[26] Puttamadappa, C., and B. D. Parameshachari. "Demand side management of small scale loads in a smart grid using glow-worm swarm optimization technique." Microprocessors and Microsystems 71 (2019): 102886.

[27] Parameshachari, B. D., H. T. Panduranga, and Silvia liberata Ullo. "Analysis and computation of encryption technique to enhance security of medical images." In IOP Conference Series: Materials Science and Engineering, vol. 925, no. 1, p. 012028. IOP Publishing, 2020.

[28] Z. Guo, L. Tang, T. Guo, K. Yu, M. Alazab, A. Shalaginov, "Deep Graph Neural Network-based Spammer Detection Under the Perspective of Heterogeneous Cyberspace", Future Generation Computer Systems, https://doi.org/10.1016/j.future.2020.11.028.

[29] N. Shi, L. Tan, W. Li, X. Qi, K. Yu, "A Blockchain-Empowered AAA Scheme in the Large-Scale HetNet", Digital Communications and Networks, https://doi.org/10.1016/j.dcan.2020.10.0 02 .

[30] L. Tan, H. Xiao, K. Yu, M. Aloqaily, Y. Jararweh, "A Blockchain-empowered Crowdsourcing System 
for 5G-enabled Smart Cities”, Computer Standards \&

Interfaces, https://doi.org/10.1016/j.csi.2021.10351 7

[31] K. Yu, L. Tan, M. Aloqaily, H. Yang, and Y. Jararweh, "Blockchain-Enhanced Data Sharing with Traceable and Direct Revocation in IIoT", IEEE Transactions on Industrial Informatics, doi: 10.1109/TII.2021.3049141.

[32] L. Tan, N. Shi, K. Yu, M. Aloqaily, Y. Jararweh, "A Blockchain-Empowered Access Control Framework for Smart Devices in Green Internet of Things", ACM Transactions on Internet Technology, vol. 21, no. 3, pp. 1-20, 2021,https://doi.org/10.1145/3433542.

[33] L. Zhen, A. K. Bashir, K. Yu, Y. D. Al-Otaibi, C. H. Foh, and P. Xiao, "Energy-Efficient Random Access for LEO Satellite-Assisted 6G Internet of Remote Things", IEEE Internet of Things Journal, doi: 10.1109/JIOT.2020.3030856. 